\title{
Letrozole as an adjunct treatment in antagonist cycles that previously failed in poor responders
}

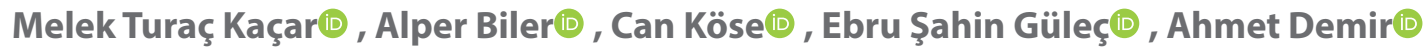 \\ University of Health Sciences Tepecik Education and Research Hospital, Izmir, Turkey
}

\begin{abstract}
Objectives: To investigate whether adding letrozole in the early follicular phase of a gonadotropin-releasing hormone $(\mathrm{GnRH})$ antagonist (GA) stimulation cycle improves in vitro fertilization (IVF) outcomes in poor responder patients.

Material and methods: To be included in this study, patients had to have had at least one previous GA cycle and a subsequent GA cycle with added early follicular phase letrozole (LzGA). A total of 41 poor responder patients were identified based on the Bologna criteria.

Results: The LzGA group had a lower dosage of follicular stimulating hormone (FSH) $(p=0.001)$, the duration of stimulation days $(p=0.015)$ and the duration of $\mathrm{GnRH}$ antagonist stimulation days $(p=0.033)$ when compared with controls. Comprehensive analysis of the cycle characteristics showed that the number of oocytes retrieved, the number of MII oocytes retrieved, the number of fertilized oocytes, and the fertilization rate were significantly higher in the LzGA cycle ( $p=0.041$, $p=0.019, p=0.008, p=0.01$, respectively). The rate of cycle cancellation was lower in the LzGA group (24.4\%) than in the GA group (48.8\%), $(p<0.001)$. Although LzGA administration demonstrated a trend toward improved implantation and clinical pregnancy rates, this was an insignificant trend $(p=1.000, p=0.177$, respectively).

Conclusions: Adjunctive letrozole administration seems to restore an IVF cycle by improving the cycle characteristics and reducing the total gonadotrophin dosage.
\end{abstract}

Key words: letrozole; ovarian stimulation; poor response; diminished ovarian reserve; antagonist cycle

Ginekologia Polska 2022; 93, 4: 267-272

\section{INTRODUCTION}

Diminished ovarian reserve (DOR) is defined as the reduced ability to achieve pregnancy and poor ovarian response to gonodotropin stimulation compared with women of a similar age [1]. In most patients, DOR remains unexplained but may be caused by advanced age, previous ovarian surgery, severe endometriosis, and environmental or genetic factors $[2,3]$. DOR is often related to poor ovarian response to standard ovulation induction protocol and manifests as poor fertility results even when assisted reproductive techniques (ART) are used. Reduced implantation rate, decreased pregnancy rate, increased gonadotrophin use, and increased cycle cancellation are some of the main challenges in these patients.

Numerous investigations have been published about the management of poor ovarian responders, and they have proposed various stimulation protocols to improve pregnancy outcomes [4-7]. Increased gonadotrophin use, gonadotropin-releasing hormone (GnRH) agonist flare-up protocols, natural cycle in vitro fertilization (IVF), and adjuvant therapies have been studied [8, 9]. Advised adjuvant therapies include growth hormone, aspirin, pyridostigmin, L-arginine, androgen supplements (testosterone and dehydroepiandrosterone), and androgen-modulating agents (letrozole and anastrozole). However, there is a lack of consensus regarding most interventions proposed to improve pregnancy rates in these patients.

Letrozole is a potent, highly selective, non-steroidal third-generation aromatase inhibitor. It prevents estrogen syntheses by inhibiting the aromatase enzyme activity. The resulting decrease in early follicular phase estrogen levels diminishes the negative feedback of estrogen on follicular stimulating hormone (FSH), consequently causing an augmentation in endogenous gonadotropin secretion and stimulation of ovarian follicular growth [10, 11]. The other proposed mechanism of action includes raised intraovarian androgens [10]. An increase in intraovarian androgens as a result of aromatase inhibition increases the expression

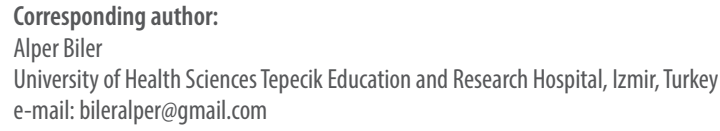


of FSH receptors on the follicle. Follicular sensitivity to FSH stimulation is thereby augmented [11].

Letrozole could successfully induce both ovulation and ovarian stimulation without any negative effects on the endometrium in women with polycystic ovary syndrome (PCOS) $[11,12]$. A few initial studies also showed that, when letrozole is added to a gonadotropin ovulation induction in poor responder patients, the ovarian response to FSH increases and the gonadotropin doses required for stimulation are decreased [13-15]. However, recent studies on the use of letrozole in this patient group have reported inconsistent results [16-21]. This study therefore set out to assess whether adding letrozole in the early follicular phase of a GnRH antagonist (GA) stimulation cycle improves IVF outcomes in poor responders.

\section{MATERIAL AND METHODS}

\section{Patient selection, stimulation protocol, oocyte retrieval and transfer}

This retrospective study was conducted at the University of Health Sciences Tepecik Education and Research Hospital IVF Centre between January 2017 and December 2018. The medical records of 246 infertile patients with DOR according to the Bologna criteria of the 2011 European Society of Human Reproduction and Embryology consensus [22] were screened. To be included in this study, patients had to have had at least one previous GA cycle and a subsequent GA cycle with added early follicular phase letrozole (LzGA). A total of 41 patients with at least one previous GA cycle followed by an LzGA cycle were identified. Each patient was included only once. Women with multiple ART cycles who had previously had a GA cycle and then had several LzGA cycles were included only for the first GA cycle and the subsequent LzGA cycle. Patients with additional infertility factors, such as PCOS, tubal factors, and male factors, and who had received the treatment cycles more than six months apart were excluded from the study. The study protocol was approved by the Institutional Review Board of the University of Health Sciences, Tepecik Education and Research Hospital, Izmir, Turkey (approval number 2018/5-11).

Eligible women were evaluated on day two or three of their menstrual cycle by transvaginal ultrasound to measure endometrial lining, perform an antral follicle count, and exclude the presence of ovarian cysts. Blood samples were also taken for serum FSH, luteinizing hormone (LH), estradiol (E2), progesterone (P), and thyroid stimulating hormone (TSH). Subsequently, in GA cycle, recombinant FSH (Gonal-F; Merck-Serono, Istanbul) and highly purified hMG (Merional; IBSA, Istanbul, Turkey) were used at doses ranging between 225 and $375 \mathrm{IU} /$ day. The dosages of FSH and hMG were adjusted according to the ovarian response. The ovarian response of patients was monitored by trans- vaginal ultrasound and serum E2 levels. A flexible $\mathrm{GnRH}$ antagonist protocol (Cetrotide, $0.25 \mathrm{mg} /$ day, Merc-Serono, Istanbul, Turkey) was initiated when the average diameter of the leading follicle was 13-14 mm and/or the serum E2 level was $>350 \mathrm{pg} / \mathrm{mL}$, and the protocol was administered until the day of human chorionic gonadotropin (hCG). Recombinant hCG $250 \mu \mathrm{g}$ (Ovitrelle; Merck-Serono, Istanbul, Turkey) was administrated to trigger follicle maturation when at least two follicles measuring $\geq 17 \mathrm{~mm}$ in diameter.

In subsequent LzGA cycles of patients, hormonal and ultrasonographic measurements were taken on day two or three of the menstrual cycle. Letrozole (Femara; Novartis, Istanbul, Turkey) at a dose of $5 \mathrm{mg} /$ day $(2.5 \mathrm{mg} \times 2)$ was initiated on day two or three and continued for five days. Ovarian stimulation, use of the $\mathrm{GnRH}$ antagonist protocol, and triggering of follicle maturation were performed, similarly to their preceding cycles. Oocyte retrieval was carried out by transvaginal ultrasonography under general anesthesia 35-36 hours after the ovulation trigger. Intracytoplasmic sperm injection (ICSI) was performed for all patients. The embryo quality was evaluated in the embryo cleavage stage (2-3 days) and morula-blastocyst stage (4-6 days) $[23,24]$. Single or double embryos were transferred between day two and five under transabdominal ultrasound guidance. The luteal phase was support with intravaginal progesterone gel (Crinone; Merc-Serono, Istanbul, Turkey) starting on the day of oocyte retrieval and continuing until the $12^{\text {th }}$ week of pregnancy in cases with positive pregnancy tests. The ß-hCG serum level was measured to confirm pregnancy on the $12^{\text {th }}$ day after embryo transfer. Pregnancy was defined as blood B-hCG $\geq 20 \mathrm{IU} / \mathrm{L}$.

\section{Pregnancy}

Clinical pregnancy was defined as presence of a fetal heartbeat detected by transvaginal ultrasound scan that was performed 4-5 weeks after embryo transfer. Implantation rate (IR) was calculated by dividing the number of gestational sacs transplanted embryos. Live birth was defined as an infant born alive after the $24^{\text {th }}$ gestational week.

\section{Outcome measurements}

The mean cumulative gonadotrophin dosage, mean duration of gonadotrophin stimulation days, mean duration of $\mathrm{GnRH}$ antagonist stimulation days, mean serum estradiol concentration on the day of hCG administration, mean number of oocytes retrieved, mean number of mature oocytes (metaphase Il oocytes), mean number of fertilized oocytes [mean number of 2 pronucleate (2PN) zygotes], fertilization rates, mean number of transferred embryos, mean number of transferred embryos for 2-3 days and 5-6 days, clinical pregnancy rates, and live birth rates were compared between patients who received the two stimulation protocols. 


\section{Statistics}

Statistical calculations were done using SPSS for Windows version 20.0 (SPSS, Chicago,USA). The mean values are expressed as mean \pm standard deviation. Statistical comparison was carried out by Student's t-test for continuous variables and the chi-square test or Fisher's exact test for categorical variables. A $p$ value of $<0.05$ was considered statistically significant.

\section{RESULTS}

A total of 41 patients, diagnosed as poor responders based on the Bologna criteria, were eligible for inclusion. A GA protocol and an LzGA protocol were applied to all patients. Table 1 shows the baseline characteristics of the study patients. Their age was $34.3 \pm 4.25$. The duration of infertility was $7.47 \pm 5.1$ years. Basal hormone levels were similar among both cycles except for E2 levels on day three (Tab. 2).

The used gonadotroin units $(2151.21 \pm 649.61$ vs $2807.62 \pm 1125.55 ; p=0.001)$, the number of stimulation days $(7.63 \pm 1.95$ vs $8.87 \pm 2.71 ; p=0.015)$ and the duration of $\mathrm{GnRH}$ antagonist stimulation days ( $3.97 \pm 1.42$ vs $4.80 \pm 1.92$; $\mathrm{p}=0.033$ ) were significantly lower in the LzGA cycle than in the GA cycle (Tab. 3). Although the mean number of follicles on the trigger day were similar in both cycles, the mean number of retrieved oocytes and the number of metaphase II oocytes were significantly higher in LzGA (Tab. 3). The mean peak E2 level on the trigger day was also found to be significantly lower in the LzGA cycle than in the GA cycle (Tab. 3). LzGA had a higher number of fertilized oocytes

\begin{tabular}{|l|c|}
\hline \multicolumn{2}{|l|}{ Table 1. Demographic characteristics of patients } \\
\hline \\
\hline Age, years (mean \pm SD) & $\mathbf{n : 4 1}$ \\
\hline $\mathrm{BMI}, \mathrm{kg} / \mathrm{m}^{2}$ (mean $\pm \mathrm{SD}$ ) & $34.36 \pm 4.25$ \\
\hline Gravida (mean $\pm \mathrm{SD}$ ) & $25.12 \pm 4.31$ \\
\hline Parity (mean $\pm \mathrm{SD}$ ) & $0.24 \pm 0.43$ \\
\hline Live birth number (mean \pm SD) & 0 \\
\hline Abortion (mean \pm SD) & 0 \\
\hline Menstruel cycle duration (mean $\pm \mathrm{SD}$ ) & $0.17 \pm 0.38$ \\
\hline Male age, years (mean $\pm \mathrm{SD}$ ) & $26.26 \pm 4.82$ \\
\hline Duration of infertility, years (mean \pm SD) & $37.36 \pm 5.26$ \\
\hline
\end{tabular}

Values are mean $\pm \mathrm{SD} ; \mathrm{SD}$ — standard deviation; $\mathrm{BMI}$ — body mass index

Table 2. Basal hormone concentrations in cycles letrozole $+\mathrm{GnRH}$ antagonist and $\mathrm{GnRH}$ antagonist

\begin{tabular}{|l|c|c|c|}
\hline & LzGA cycle & GA cycle & p \\
\hline Day-3 serum FSH $[\mathrm{mlU} / \mathrm{mL}]$ & $12.82 \pm 4.38$ & $11.24 \pm 5.31$ & 0.094 \\
\hline Day-3 serum LH [mIU/mL] & $5.23 \pm 3.49$ & $4.64 \pm 2.43$ & 0.223 \\
\hline Day-3 serum E2 [pg/mL] & $49.97 \pm 23.78$ & $39.69 \pm 20.01$ & 0.014 \\
\hline Day-3 serum progesterone $[\mathrm{ng} / \mathrm{mL}]$ & $0.82 \pm 0.58$ & $0.79 \pm 0.49$ & 0.763 \\
\hline TSH [mlU/L] & $1.52 \pm 0.75$ & $1.49 \pm 0.85$ & 0.832 \\
\hline Prolactine $[\mathrm{ng} / \mathrm{mL}]$ & $14.89 \pm 5.95$ & $16.56 \pm 8.10$ & 0.060 \\
\hline AMH $[\mathrm{ng} / \mathrm{mL}]$ & $0.68 \pm 0.33$ & $0.68 \pm 0.34$ & 0.323 \\
\hline Antral follicle count $(\mathrm{n})$ & $4.5 \pm 2.73$ & $3.9 \pm 2.42$ & 0.276 \\
\hline
\end{tabular}

Values are mean $\pm \mathrm{SD} ; \mathrm{p}$ value $\leq 0.05$ was considered statistically significant; FSH — follicle stimulating hormone; LH — luteinizin hormone; E2 — estradiol; TSH — tiroid stimulating hormone; AMH — anti-mullerian hormone; LzGA — letrozole + GnRH antagonist; GA — GnRH antagonist

\begin{tabular}{|c|c|c|c|}
\hline n: 41 & LzGA cycle & GA cycle & $\mathbf{p}$ \\
\hline Total gonadotrophin consumption [IU] & $2151.21 \pm 649.61$ & $2807.62 \pm 1125.55$ & 0.001 \\
\hline Duration of stimulation [day] & $7.63 \pm 1.95$ & $8.87 \pm 2.71$ & 0.015 \\
\hline Duration of $\mathrm{GnRH}$ antagonist stimulation [day] & $3.97 \pm 1.42$ & $4.80 \pm 1.92$ & 0.033 \\
\hline Number of follicles on day of hCG & $2.70 \pm 2.13$ & $2.43 \pm 1.94$ & 0.572 \\
\hline Oocytes retrieved (n) & $2.82 \pm 1.37$ & $2.14 \pm 0.041$ & 0.041 \\
\hline MIl oocytes retrieved $(n)$ & $2.56 \pm 1.46$ & $1.85 \pm \underline{? ? ?}$ & 0.019 \\
\hline Peak E2 level [pg/mL] & $557.43 \pm 403.58$ & $776.24 \pm 376.46$ & 0.002 \\
\hline Fertilized oocytes (n) & $1.82 \pm 1.37$ & $1.19 \pm 1.32$ & 0.008 \\
\hline Fertilization rate [\%] & $68.55 \pm 35.21$ & $49.75 \pm 44.86$ & 0.01 \\
\hline
\end{tabular}

Values are mean \pm SD; $p$ value $\leq 0.05$ was considered statistically significant; $G n R H$ - gonadotropin releasing hormone; $h C G$ — human chorionic gonadotropin; MII — metaphase II; E2 - estradiol; LzGA — letrozole + GnRH antagonist; GA — GnRH antagonist 
and fertilization rate $(1.82 \pm 1.37$ vs $1.19 \pm 1.32 ; p=0.008$, $68.55 \pm 35.21$ vs $49.75 \pm 44.86 ; p=0.01$, respectively) (Tab. 3 ). In the GA cycle, the cancellation rate was $48.8 \%$, whereas in the LzGA cycle, it was $24.4 \%$ ( $p<0.001$ ). The causes of cancellation are summarized in Table 4. The day on which embryos were transferred and the pregnancy results are shown Table 5. Seven clinical pregnancies (17.07\%) in the LzGA group and three clinical pregnancies (7.3\%) in the GA group were recorded. Although LzGA administration was 2.6 times more common in clinical pregnancy when compared with GA administration, this was a non-significant trend toward higher clinical pregnancy rates in LzGA administration. Of the seven patients who had clinical pregnancy after LzGA administration, four patients (9.75\%) had a live birth.

\section{DISCUSSION}

Our results show that, when compared with the previous GA cycle, the succeeding LzGA cycle resulted in a significant reduction in the gonadotrophin dose needed for ovarian stimulation (OS) and a reduction of the number of stimulation days and the number of GnRH antagonist stimulation days. Comprehensive analysis of the cycle characteristics showed that the number of oocytes, Mll oocytes, and fertilized oocytes retrieved, as well as the fertilization rate, were significantly higher in the LzGA cycle. Moreover, lower cancellation rates were observed when adding letrozole to subsequent GnRH antagonist cycles. Letrozole's positive effects on cycle characteristics, implantation rates,

\begin{tabular}{|c|c|c|c|}
\hline & LzGA & GA & p \\
\hline Cycle cancellation rate $\mathrm{n},(\%)$ & $10(24.4)$ & $20(48.8)$ & $<0.001$ \\
\hline \multicolumn{4}{|l|}{ Causes of cansellation $(n=30)$} \\
\hline Total fertilization failure & 4 & 10 & \\
\hline No oocyte in the OPU & 2 & 5 & \\
\hline Arrest of embryo growth & 3 & 1 & \\
\hline Poor morphology & 1 & 4 & \\
\hline
\end{tabular}

$\mathrm{OPU}$ - oocyte pick-up; LzGA - letrozole + GnRH antagonist; GA - GnRH antagonist

\begin{tabular}{|l|c|c|c|}
\hline \multicolumn{4}{|c|}{ Table 5. Days of transferred embryos and pregnancy rates } \\
\hline & LzGA cycle & GA cycle & p \\
\hline Day 2-3 embryo transfer, $\mathrm{n}(\%)$ & $28(68.3)$ & $19(39.0)$ & 0.015 \\
\hline Day 5 embryo transfer, $\mathrm{n}(\%)$ & $3(7.3)$ & $2(4.9)$ & 1.000 \\
\hline Clinical pregnancyn (\%) & $7(17.07)$ & $3(7.3)$ & 0.177 \\
\hline Implantation rate, $\mathrm{n}(\%)$ & $7(17.07)$ & $6(12.5)$ & 1.000 \\
\hline Live births, $\mathrm{n}(\%)$ & $4(9.75)$ & $0(0)$ & 0.124 \\
\hline
\end{tabular}

LzGA - letrozole + GnRH antagonist; GA — GnRH antagonist and clinical pregnancy rates between cycles were not statistically different.

Low response to OS, increased gonadotrophin use, reduced implantation rate, and decreased prospects of pregnancy are the clinical signs of DOR. The administration of ART cycles for these patient populations is one of the largest difficulties for the clinician. Although there is not a strong relationship between pregnancy results and the use of letrozole in the first days of the follicular phase of OS in poor responder patients, various positive effects on the cycles have been found [16-21, 25-27]. In a randomized study conducted by Ozmen et al. [21], ovarian stimulation with FSH plus letrozole along with GnRH antagonist in poor responder patients significantly reduces the necessary doses of gonadotrophin and the cost of gonadotrophin stimulation. More recently, Lee et al. [26], analyzed a total of 103 consecutive IVF cycles in poor responder patients performed with either FSH plus letrozole along with $\mathrm{GnRH}$ antagonist or with only FSH along with $\mathrm{GnRH}$ antagonist. They reported that the total doses of gonadotrophin and days of gonadotrophin administration were significantly lower in the letrozole group. In addition, the GnRH antagonist administration days was significantly decreased in the letrozole group in this study. A possible explanation is that letrozole increases ovarian sensitivity to gonadotrophins, and consequently, gonadotropin consumption and the duration of stimulation days decrease. In the present study, we demonstrated that the addition of letrozole to the early follicular phase of an OS cycle significantly reduced gonadotrophin consumption and the duration of the cycle, which is consistent with the above-mentioned studies. The GnRH antagonist stimulation days was also shorter in the letrozole group.

When the cycle characteristics are examined, the results of the use of letrozole in the early follicular phase and in previous studies are slightly more conflicting [17, 18, 21, 25-27]. Ozmen et al., Goswmi et al., and Ebrahimi et al., reported a comparable number of retrieved oocytes in the letrozole group, whereas Lee VC et al., Lee KH et al., and Garcio-Velasco et al., reported a significantly higher number of oocytes retrieved [17, 18, 21, 25-27]. Consistently with the studies performed by Lee VC et al., Lee KH et al., and Garcio-Velasco et al., the present research demonstrated that a significantly increased number of oocytes were retrieved in the LzGA cycle $[25,26,17]$. The number of metaphase Il oocytes was significantly higher, consistent with the total number of oocytes retrieved. Similarly, both Ozmen et al., and Ebrahimi et al., found the number of metaphase II oocytes to be consistent with the total number of oocytes retrieved [21, 27]. However, in the study of Lee et al. [26], the number of oocytes retrieved and the number of Mll oocytes were not consistent. 
IVF treatment results can be expected to improve when significantly more oocytes are collected and more MII oocytes are obtained, probably because they augment embryo selection for embryo transfer [28, 29]. However, the most previous studies showed similar IVF outcomes in the letrozole group. [21, 25-27]. Pregnancy results in the letrozole group did not differ significantly in any of these studies. Ebrahimi et al. [27], added letrozole to a stimulation program for poor responder patients, identified based on the Bologna criteria, and there were no significant differences between groups regarding the number of oocytes retrieved, fertilization rate, implantation rate, total cycle cancelation rate, and clinical pregnancy rate. They suggested that the use of letrozole does not improve clinical outcomes in poor responder patients. A randomized, controlled trial conducted by Goswami et al. [18], reported similar numbers of oocytes retrieved and pregnancy rates between groups, except for the group with a significantly lower total dose of FSH. These results were consistent with what has been stated in various studies [21, 25-27]. Moreover, some studies had inconsistencies within themselves $[17,30]$. In the Garcia-Velasco study [17], evaluating the impact of letrozole as an adjuvant treatment in IVF cycles on low responder patients, there was a significant improvement in the implantation rate and the number of oocytes retrieved in the group with added letrozole, but there was no significant difference between the groups regarding cycle cancelation, fertilization, or pregnancy rates. More recently, Moinid et al. [30], compared letrozole plus GnRH antagonist with a placebo plus $\mathrm{GnRH}$ antagonist in poor responders, and they showed that the total number of retrieved oocytes and of Mll oocytes in the letrozole-treated group were significantly higher than in the control group. However, there were no marked differences regarding fertilization rate, implantation rate, or clinical pregnancy. In the current study, implantation rates and clinical pregnancy rates were comparable between groups despite the higher number of retrieved oocytes, MII oocytes, and fertilized oocytes, the increased fertilization rate, and the lower cycle cancellation rates in patients receiving letrozole. These results were in accordance with previous studies $[17,30]$.

In light of these findings, positive results of studies should be evaluated with caution because ovarian responses depend on cyclic fluctuations, and patients with a poor response in the first cycle might respond normally in the subsequent cycle [31]. Therefore, improvement in cycle results with letrozole might be linked to the fluctuation in ovarian response, not to the drug's effect. On the other hand, by blocking the conversion of androstenedione and testosterone to estrogen, letrozole might increase endogenous gonadotropin secretion and stimulate ovarian follicular growth, leading to a chance to produce more oocytes $[10,11]$. The outcomes of the present study support the literature that letrozole might improve the cycle characteristics and comparable pregnancy results in poor responder patients.

The main criticism of the current study are its retrospective nature and small sample size. Retrospective cohort studies are subject to selection bias, recall bias, and unknown confounding variables, which may negatively affect the accuracy of the results. Therefore, the results of the current study need to be interpreted carefully until well-designed, prospective randomized trials have been performed.

\section{CONCLUSIONS}

Our findings indicate that adding letrozole in the early follicular phase of a GnRH antagonist stimulation cycle has benefits in reducing the required dose of gonadotrophin and in improving the success of cycle characteristics in poor responders. Moreover, while there is a trend toward improved implantation and clinical pregnancy rates, these results are not statistically significant. Nevertheless, it is impossible to say that using letrozole as an adjuvant agent has no positive effects in poor responder patients during the stimulation cycle. However, further randomized controlled trials are required to confirm these findings.

\section{Conflict of interest}

The authors declare that they have no conflict of interest.

\section{REFERENCES}

1. Practice Committee of the American Society for Reproductive Medicine. Testing and interpreting measures of ovarian reserve: a committee opinion. Fertil Steril. 2015; 103(3): e9-ee17, doi: 10.1016/j.fertnstert.2014.12.093, indexed in Pubmed: 25585505.

2. Toner J, Philput $C$, Jones $\mathrm{G}$, et al. Basal follicle-stimulating hormone level is a better predictor of in vitro fertilization performance than age ${ }^{* *}$ Presented as the Prize Paper for the Society of Assisted Reproductive Technologies at the Annual Meeting of The American Fertility Society, Washington, D.C., October 15 to 18, 1990. Fertility and Sterility. 1991; 55(4): 784-791, doi: 10.1016/s0015-0282(16)54249-6, indexed in Pubmed: 1901282.

3. Wardle PG, Mclaughlin EA, Mcdermott A, et al. ENDOMETRIOSIS AND OVULATORY DISORDER: REDUCED FERTILISATION IN VITRO COMPARED WITH TUBAL AND UNEXPLAINED INFERTILITY. The Lancet. 1985; 326(8449): 236-239, doi: 10.1016/s0140-6736(85)90289-2, indexed in Pubmed: 2862417.

4. Fasouliotis SJ, Simon A, Laufer N. Evaluation and treatment of low responders in assisted reproductive technology: a challenge to meet. J Assist Reprod Genet. 2000; 17(7):357-373, doi: 10.1023/a:1009465324197, indexed in Pubmed: 11077616.

5. Mahutte NG, Arici A. Poor responders: does the protocol make a difference? Curr Opin Obstet Gynecol. 2002; 14(3): 275-281, doi: 10.1097/00001703-200206000-00005, indexed in Pubmed: 12032382.

6. Surrey E, Schoolcraft W. Evaluating strategies for improving ovarian response of the poor responder undergoing assisted reproductive techniques. Fertility and Sterility. 2000; 73(4): 667-676, doi: 10.1016/s00150282(99)00630-5, indexed in Pubmed: 10731523.

7. Tarlatzis BC, Zepiridis L, Grimbizis G, et al. Clinical management of low ovarian response to stimulation for IVF: a systematic review. Hum Reprod Update. 2003; 9(1): 61-76, doi: 10.1093/humupd/dmg007, indexed in Pubmed: 12638782.

8. Ubaldi FM, Rienzi L, Ferrero $S$, et al. Management of poor ovarian responders in IVF. Reproductive BioMedicine Online. 2005; 10(2): 235-246, doi: 10.1016/s1472-6483(10)60946-7, indexed in Pubmed: 15823231. 
9. Pandian Z, McTavish AR, Aucott L, et al. Interventions for 'poor responders' to controlled ovarian hyper stimulation $(\mathrm{COH})$ in in-vitro fertilisation (IVF). Cochrane Database Syst Rev. 2010(1): CD004379, doi: 10.1002/14651858.CD004379.pub3, indexed in Pubmed: 20091563.

10. Akhtar M, Njar V, Wright JN. Mechanistic studies on aromatase and related $\mathrm{C} \otimes C$ bond cleaving P-450 enzymes. The Journal of Steroid Biochemistry and Molecular Biology. 1993; 44(4-6): 375-387, doi: 10.1016/0960-0760(93)90241-n, indexed in Pubmed: 8476751.

11. Mitwally MFM, Casper RF, Diamond MP. The role of aromatase inhibitors in ameliorating deleterious effects of ovarian stimulation on outcome of infertility treatment. Reprod Biol Endocrinol. 2005; 3: 54, doi: 10.1186/1477-7827-3-54, indexed in Pubmed: 16202169.

12. Mitwally M, Casper R. Use of an aromatase inhibitor for induction of ovulation in patients with an inadequate response to clomiphene citrate. Fertility and Sterility. 2001; 75(2): 305-309, doi: 10.1016/s00150282(00)01705-2, indexed in Pubmed: 11172831.

13. Mitwally $M$, Casper R. Aromatase inhibition improves ovarian response to follicle-stimulating hormone in poor responders 1 1Recipient of the 2001 Pacific Coast Reproductive Society-Wyeth-Ayerst Award. Fertility and Sterility. 2002; 77(4): 776-780, doi: 10.1016/s0015-0282(01)03280-0, indexed in Pubmed: 11937133

14. Mitwally MFM, Casper RF. Aromatase inhibitors for the treatment of infertility. Expert Opin Investig Drugs. 2003; 12(3): 353-371, doi: 10.1517/13543784.12.3.353, indexed in Pubmed: 12605560.

15. Healey S, Tan SL, Tulandi T, et al. Effects of letrozole on superovulation with gonadotropins in women undergoing intrauterine insemination. Fertil Steril. 2003; 80(6): 1325-1329, doi: 10.1016/j.fertnstert.2003.03.001, indexed in Pubmed: 14667860

16. Davar R, Oskouian H, Ahmadi S, et al. GnRH Antagonist/Letrozole Versus Microdose GnRH Agonist Flare Protocol in Poor Responders Undergoing In Vitro Fertilization. Taiwanese Journal of Obstetrics and Gynecology. 2010; 49(3): 297-301, doi: 10.1016/s1028-4559(10)60064-2, indexed in Pubmed: 21056314.

17. Garcia-Velasco JA, Moreno L, Pacheco A, et al. The aromatase inhibitor letrozole increases the concentration of intraovarian androgens and improves in vitro fertilization outcome in low responder patients: a pilot study. Fertil Steril. 2005; 84(1): 82-87, doi: 10.1016/j.fertnstert.2005.01.117, indexed in Pubmed: 16009161.

18. Goswami SK, Das T, Chattopadhyay R, et al. A randomized single-blind controlled trial of letrozole as a low-cost IVF protocol in women with poor ovarian response: a preliminary report. Hum Reprod. 2004; 19(9): 2031-2035, doi: 10.1093/humrep/deh359, indexed in Pubmed: 15217999.

19. Schoolcraft WB, Surrey ES, Minjarez DA, et al. Management of poor responders: can outcomes be improved with a novel gonadotropin-releasing hormone antagonist/letrozole protocol? Fertil Steril. 2008; 89(1): 151-156, doi: 10.1016/j.fertnstert.2007.02.013, indexed in Pubmed: 17482177

20. Yarali $\mathrm{H}$, Esinler I, Polat $\mathrm{M}$, et al. Antagonist/letrozole protocol in poor ovarian responders for intracytoplasmic sperm injection: a comparative study with the microdose flare-up protocol. Fertil Steril. 2009; 92(1):
231-235, doi: 10.1016/j.fertnstert.2008.04.057, indexed in Pubmed: 18678368 .

21. Ozmen B, Sönmezer M, Atabekoglu CS, et al. Use of aromatase inhibitors in poor-responder patients receiving $\mathrm{GnRH}$ antagonist protocols. Reprod Biomed Online. 2009; 19(4): 478-485, doi: 10.1016/j.rbmo.2009.05.007, indexed in Pubmed: 19909587.

22. Ferraretti AP, La Marca A, Fauser BC, et al. ESHRE working group on Poor Ovarian Response Definition. ESHRE consensus on the definition of'poor response' to ovarian stimulation for in vitro fertilization: the Bologna criteria. Hum Reprod. 2011; 26(7): 1616-1624, doi: 10.1093/humrep/der092, indexed in Pubmed: 21505041.

23. Hardarson T, Hanson C, Sjögren A, et al. Human embryos with unevenly sized blastomeres have lower pregnancy and implantation rates: indications for aneuploidy and multinucleation. Hum Reprod. 2001; 16(2): 313-318, doi: 10.1093/humrep/16.2.313, indexed in Pubmed: 11157826.

24. Alpha Scientists in Reproductive Medicine and ESHRE Special Interest Group of Embryology. The Istanbul consensus workshop on embryo assessment: proceedings of an expert meeting. Hum Reprod. 2011; 26(6): 1270-1283, doi: 10.1093/humrep/der037, indexed in Pubmed: 21502182.

25. Lee VC, Chan $\mathrm{CC}, \mathrm{Ng} E \mathrm{EH}$, et al. Sequential use of letrozole and gonadotrophin in women with poor ovarian reserve: a randomized controlled trial. Reprod Biomed Online. 2011; 23(3): 380-388, doi: 10.1016/j. rbmo.2011.05.012, indexed in Pubmed: 21782514.

26. Lee $\mathrm{KH}, \mathrm{Kim} \mathrm{CH}$, Suk HJ, et al. The effect of aromatase inhibitor letrozole incorporated in gonadotrophin-releasing hormone antagonist multiple dose protocol in poor responders undergoing in vitro fertilization. Obstet Gynecol Sci. 2014; 57(3): 216-222, doi: 10.5468/ogs.2014.57.3.216, indexed in Pubmed: 24883293.

27. Ebrahimi M, Akbari-Asbagh F, Ghalandar-Attar M, et al. Letrozole+ GnRH antagonist stimulation protocol in poor ovarian responders undergoing intracytoplasmic sperm injection cycles: An RCT. Int J Reprod Biomed. 2017 15(2): 101-108, doi: 10.29252/ijrm.15.2.101, indexed in Pubmed: 28462402.

28. Sunkara SK, Rittenberg V, Raine-Fenning N, et al. Association between the number of eggs and live birth in IVF treatment: an analysis of 400135 treatment cycles. Hum Reprod. 2011; 26(7): 1768-1774, doi: 10.1093/humrep/der106, indexed in Pubmed: 21558332.

29. Samara N, Reis D, Danielli Miller N, et al. What are the best predictors for successful $\mathrm{GnRH}$ antagonist protocol in in vitro fertilization (IVF) treatment? Gynecol Endocrinol. 2015; 31(11): 877-879, doi: 10.3109/09513590.2015.1081680, indexed in Pubmed: 26416777.

30. Moini A, Lavasani Z, Kashani L, et al. Letrozole as co-treatment agent in ovarian stimulation antagonist protocol in poor responders: A double-blind randomized clinical trial. Int J Reprod Biomed. 2019; 17(9): 653-660, doi: 10.18502/ijrm.v17i9.5101, indexed in Pubmed: 31646260.

31. Klinkert ER, Broekmans FJM, Looman CWN, et al. A poor response in the first in vitro fertilization cycle is not necessarily related to a poor prognosis in subsequent cycles. Fertil Steril. 2004; 81(5): 1247-1253, doi: 10.1016/j.fertnstert.2003.10.030, indexed in Pubmed: 15136085. 